Supramolecular Chemistry

DOI: 10.1002/ange.200504312

\section{Polymer-Induced Heteronucleation for the Discovery of New Extended Solids**}

\author{
Adam L. Grzesiak, Fernando J. Uribe, \\ Nathan W. Ockwig, Omar M. Yaghi, and \\ Adam J. Matzger*
}

The assembly of components from solution into the solid state can be accomplished in numerous manners, and each of these pathways can give rise to unique materials. The effects of the assembly pathway can be relatively subtle, as in the crystallization of pharmaceuticals into polymorphic crystals, ${ }^{[1]}$ or more dramatic, involving changes in strong bonding, as is often encountered in the preparation of extended solids. ${ }^{[2]}$ In general, there is no explicit connection between the growth conditions and the structure of the resultant phase. Indeed, spectacular changes in structure can arise from subtle differences in experimental conditions. Recently, we have introduced the use of heterogeneous nucleation from insoluble polymers as a powerful technique for efficiently discovering and selecting among different polymorphic forms of pharmaceuticals. ${ }^{[3]}$ It is proposed that the polymer serves to direct the formation of one phase over others at the time of nucleation by selectively stabilizing one mode of aggregation under conditions (solvent, temperature, extent of supersaturation, etc.) that are otherwise identical. We sought to examine the applicability of this technique towards the emergent area of metal-organic frameworks (MOFs), where it is highly desirable to establish a relationship between the crystallization conditions and the outcome of the MOF synthesis. Herein, we show that polymer substrates of varying functionality can be successfully employed to access MOF structures that otherwise are not obtainable.

We chose, as initial objects of study, MOF-2 and MOF-5 (Figure 1), two of the early reported MOFs based on the wellstudied system of 1,4-benzenedicarboxylate (BDC) and $\mathrm{Zn}^{2+}$ ions. Either paddle-wheel (MOF-2) or basic zinc acetate (MOF-5) secondary building units (SBUs) are interconnected to produce square-grid or primitive-cubic framework structures, respectively. ${ }^{[2 \mathrm{~d}, \mathrm{e}]}$ It was anticipated that, in analogy to the phase selection obtained for polymorphic pharmaceut-

[*] A. L. Grzesiak, F. J. Uribe, N. W. Ockwig, Prof. O. M. Yaghi, Prof. A. J. Matzger

Department of Chemistry

University of Michigan

Ann Arbor, MI 48109-1055 (USA)

Fax: (+1) 734-615-8853

E-mail: matzger@umich.edu

[***] This work was supported by the U.S. Department of Energy and the Beckman Foundation. A.L.G. was a fellow of the NSF-sponsored IGERT program for Molecularly Designed Electronic, Photonic, and Nanostructured Materials at the University of Michigan.

$\square$ Supporting information for this article is available on the WWWW under http://www.angewandte.org or from the author.
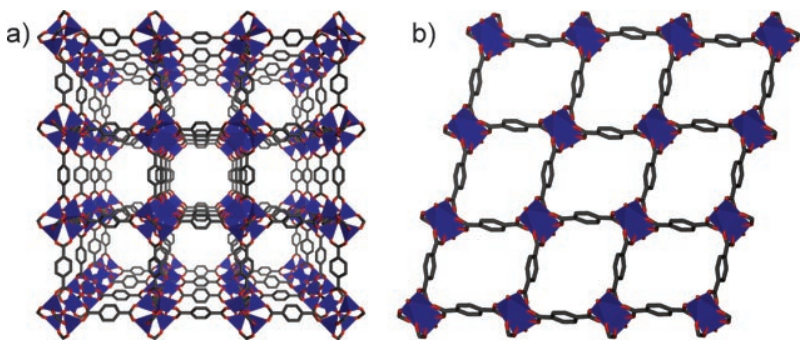

Figure 1. Packing diagrams of a) MOF-5 and b) MOF-2, which are derived from identical synthetic components, but have different crystal structures. Dark blue polyhedra represent the coordination environments of the $\mathrm{Zn}$ atoms; $\mathrm{C}$ gray, $\mathrm{O}$ red.

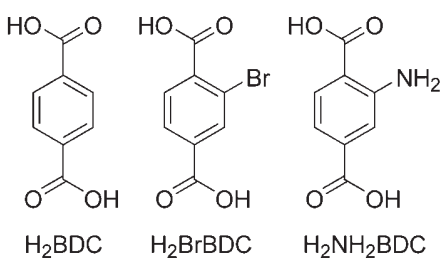

icals, ${ }^{[3]}$ selection between these two extended solids might be achieved under a single set of conditions by changing only the identity of a polymer heteronucleant present during network formation.

In initial experiments, the composition of the polymer substrate onto which crystallization occurs was varied to study MOF phase selection in the presence of acidic or basic functional groups. Such substrates could interact with the reactants, by hydrogen bonding to the MOF linkers or by direct coordination to the metal, to initiate crystal growth. The monomers methacrylic acid (MAA) and 4-vinylpyridine (4VP) were each individually cross-linked with divinylbenzene (DVB) in various ratios (see Experimental Section) to

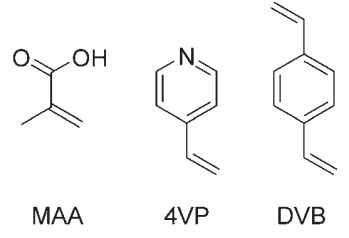

create insoluble copolymers with discrete percentages of acidic or basic functionality. This approach allows systematic exploration of the effect of substrate composition. Reagents dissolved in $N, N$-diethylformamide (DEF) were loaded into the wells of a microtiter plate, each of which contained a polymer of discrete composition that is completely insoluble, owing to extensive cross-linking. Utilizing cross-linked polymers assures a heteronucleation process and also facilitates high-throughput screening by optical microscopy. Secondary screening carried out by vibrational spectroscopy and powder X-ray diffraction (PXRD) provides unambiguous phase characterization.

To explore phase selection between MOF-2 and MOF-5, crystallization experiments were conducted with solvent and concentration conditions which were optimized for the syn- 
thesis of MOF-5 (see Experimental Section). Under these conditions, but with the addition of insoluble copolymers of MAA/DVB, both MOF-5 and an additional phase, identified as distinct from MOF-2, were formed. The new phase, designated $\quad$ PNMOF-1 $\quad($ PNMOF $=$ polymer-nucleated metal-organic framework), has a plate-like morphology and was observed in the presence of MAA/DVB copolymers with less than $30 \%$ MAA. $^{[4]}$ This phase is readily distinguished from MOF-5 by its characteristic morphology, Raman spectrum, and PXRD pattern (see Supporting Information). Its structure was determined by single-crystal X-ray diffraction, and a formula of $\mathrm{Zn}_{3}(\mathrm{BDC})_{3}(\mathrm{DEF})_{2}$ was found for the framework. ${ }^{[5]}$ The structure of PNMOF-1 is composed of trinuclear zinc carboxylate clusters joined through linear BDC linkers (Figure 2a). The clusters consist of a single octahedrally coordinated zinc center surrounded by two peripheral tetrahedrally coordinated zinc centers. One peripheral zinc center is bound to the central zinc atom through three bridging carboxylate groups. The other peripheral zinc center is bound to the central zinc atom through two bridging carboxylate groups and a single $\mu_{2}$-bridging carboxylate oxygen atom. Finally, the tetrahedral coordination sphere of the peripheral zinc centers is completed by the coordination of DEF oxygen atoms. Each of these clusters is joined to six adjacent clusters through the linear BDC linkers to yield an overall 2D triangular-grid structure containing cavities large enough to include additional solvent molecules.

PNMOF-1 was also found in the majority of crystallizations employing polymers containing $5-25 \%$ of base-functionalized 4VP cross-linked with DVB. This observation and the appearance of the PNMOF-1 phase with lower percentages of acid-functionalized MAA in the MAA/DVB polymers indicate that more nonpolar surfaces, rather than acidic or basic functionalities, facilitate the formation of PNMOF-1. To confirm this hypothesis, crystals were grown in polypropylene vessels, and PNMOF-1 was observed as the major product (see Supporting Information).

One of the outstanding features of MOFs, particularly in the isoreticular MOF (IRMOF) series, of which MOF-5 is the parent member, is the possibility of tuning their properties by altering the functionality and size of the linker ${ }^{[6]}$ Therefore, with the aim of producing additional networks with the same topology as PNMOF-1, a bromine-substituted 1,4-benzenedicarboxylic acid $\left(\mathrm{H}_{2} \mathrm{BrBDC}\right)$ was employed as the linking component in the MOF synthesis. In the absence of added polymers, the crystalline product is the cubic IRMOF-2, ${ }^{[6]}$ which is topologically identical to MOF-5. However, when the synthesis is conducted in the presence of cross-linked polymers, an additional morphologically distinct MOF phase is formed. This new phase, termed PNMOF-2, has a plate-like morphology, as well as a unique Raman spectrum and PXRD pattern (see Supporting Information). Crystals of PNMOF-2 were routinely observed in the presence of polymers containing up to $20 \%$ MAA or $30 \% 4 \mathrm{VP}$ crosslinked with DVB. The structure of PNMOF-2, as determined by single-crystal X-ray diffraction, ${ }^{[5]}$ is monoclinic and, surprisingly, has a different topology than PNMOF-1 (Figure $2 b)$. The framework of PNMOF-2 has a formula of $\left(\mathrm{Zn}_{3} \mathrm{O}\right)(\mathrm{BrBDC})_{2}\left(\mathrm{H}_{2} \mathrm{BrBDC}\right)(\mathrm{DEF})_{4}$, and is built from zinc- a)
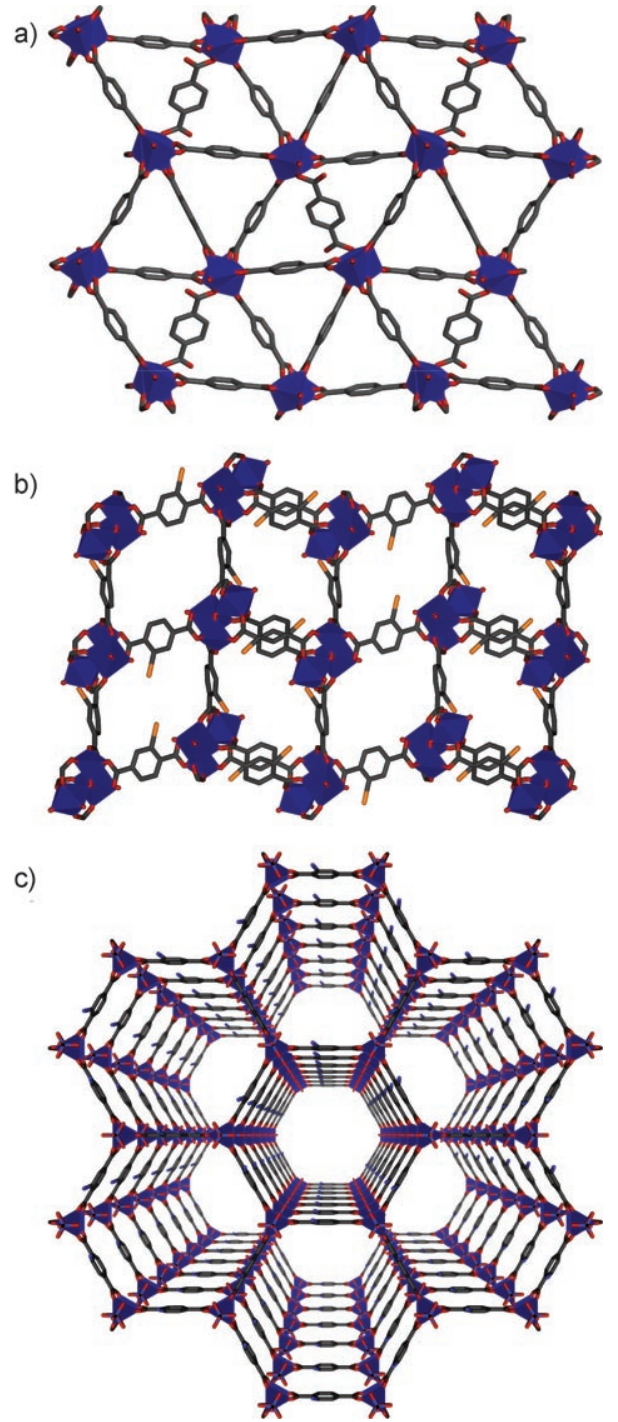

Figure 2. Crystal structures of a) PNMOF-1 $\left(\mathrm{Zn}_{3}(\mathrm{BDC})_{3}(\mathrm{DEF})_{2}\right)$, b) PNMOF-2 $\left(\left(\mathrm{Zn}_{3} \mathrm{O}\right)(\mathrm{BrBDC})_{2}\left(\mathrm{H}_{2} \mathrm{BrBDC}\right)(\mathrm{DEF})_{4}\right)$, and c) PNMOF-3 $\left(\mathrm{Zn}_{4}\left(\mathrm{NH}_{2} \mathrm{BDC}\right)_{3}\left(\mathrm{NO}_{3}\right)_{2}\left(\mathrm{H}_{2} \mathrm{O}\right)_{2}\right)$. Solvent molecules and hydrogen atoms are omitted for clarity, and disordered atoms are shown in their highest-occupancy positions. Dark blue polyhedra represent the coordination environments of the $\mathrm{Zn}$ atoms; $\mathrm{C}$ gray, $\mathrm{O}$ red, $\mathrm{N}$ light blue, Br yellow.

oxo clusters consisting of one tetrahedrally and two octahedrally coordinated zinc centers. The three zinc centers are bridged by the carboxylate groups of four BrBDC linkers, which extend the structure in three dimensions. The remaining zinc coordination sites are occupied by four oxygen atoms of terminal DEF molecules, one carbonyl oxygen atom of a linking diacid, and one $\mu_{3}$-bridging oxide anion. As in the case of PNMOF-1, PNMOF-2 grows in larger quantities from relatively more nonpolar surfaces. A common theme in these structures is the presence of DEF solvent molecules bound to zinc atoms, which perhaps favors nucleation from relatively nonpolar surfaces.

To further explore the generality of the polymer-induced heteronucleation approach to the discovery of new MOF phases, an amine-substituted dicarboxylic acid $\left(\mathrm{H}_{2} \mathrm{NH}_{2} \mathrm{BDC}\right)$ was employed as a component in the MOF synthesis. This 
relatively more polar linking unit is found in IRMOF- $3,{ }^{[6]}$ a network isostructural to MOF-5. Crystallization from conditions identical to those employed above, with the exception of a higher temperature, results in the cubic phase IRMOF-3. However, the new phase PNMOF-3 is additionally observed in crystallizations containing cross-linked MAA/DVB copolymers, but is not observed when $4 \mathrm{VP} / \mathrm{DVB}$ copolymers or polydivinylbenzene are utilized. PNMOF-3 is readily recognized because its crystals express hexagonal faces and are distinct from the crystals of the cubic IRMOF-3 phase. The crystal structure of PNMOF-3 reveals a framework of composition $\mathrm{Zn}_{4}\left(\mathrm{NH}_{2} \mathrm{BDC}\right)_{3}\left(\mathrm{NO}_{3}\right)_{2}\left(\mathrm{H}_{2} \mathrm{O}\right)_{2} \quad$ (Figure 2c). ${ }^{[5]}$ The structure is composed of trigonal clusters, in which two zinc atoms are bridged by the carboxylate functionalities of three $\mathrm{NH}_{2} \mathrm{BDC}$ linkers. The remaining coordination site of each zinc atom is occupied by either a nitrate anion or a water molecule (statistically disordered) to maintain the charge neutrality of the structure. The clusters are assembled through linear $\mathrm{NH}_{2} \mathrm{BDC}$ linkers to generate hexagonal-grid layers that are arranged in registry, yielding unusually large infinite channels of $14.9 \AA$ diameter, ${ }^{[7]}$ which are orthonormal to the layer and are occupied by crystallographically unresolved solvent molecules (see Experimental Section).

The production of PNMOF-3, in contrast to the cases of PNMOF-1 and PNMOF-2, requires the presence of MAA in the polymer heteronucleant, which demonstrates the importance of acid functionality in the selection of this framework. An increase in the yield of PNMOF-3 to approximately $75 \%$ of the product was achieved by utilizing MAA/DVB copolymers composed of $20 \%$ MAA. This success demonstrates that suitable conditions for phase production can be found on the basis of initial combinatorial discovery experiments. Notably, in contrast to the two other new MOF phases described herein, the framework of PNMOF-3 is charged, which demonstrates that a variety of extended network types may be produced by utilizing the polymer-induced heteronucleation method.

In summary, the discovery of extended solid phases using insoluble polymer substrates has been illustrated for MOFs. In these studies, conditions that yielded isostructural phases in the absence of added polymers were used. Polymer-induced heteronucleation is a simple way to increase the diversity of conditions during the crystallization of extended networks. Furthermore, the formation of a particular phase is dependent upon the polymer composition, which allows optimized production of new phases by altering polymer polarity and functionality. Studies are ongoing to identify suitable procedures for producing larger quantities of these new MOFs to thoroughly establish their properties for gas storage and catalysis applications.

\section{Experimental Section}

Polymer synthesis: MAA and 4VP were individually copolymerized with the cross-linker DVB to create copolymers with 5, 10, 15, 20, 25, $30,40,50,60$, and $70 \%$ (by volume) acidic (MAA) or basic (4VP) monomer. All samples were irradiated with an array of four 15-watt UVA lamps for $3 \mathrm{~h}$ using $1 \mathrm{~mol} \%$ 2,2'-azobisisobutyronitrile (AIBN) as an initiator. Following irradiation, the polymers were annealed at $85^{\circ} \mathrm{C}$ in a vacuum oven $(<1$ Torr) for $4 \mathrm{~h}$ to remove residual monomer, short oligomers, and solvent molecules.

MOF synthesis: MOFs were produced by heating approximately $125 \mu \mathrm{L}$ of a DEF solution containing a dicarboxylic acid $(26.7 \mathrm{~mm})$ and $\mathrm{Zn}\left(\mathrm{NO}_{3}\right)_{2} \cdot 4 \mathrm{H}_{2} \mathrm{O} \quad(80.0 \mathrm{~mm})$ to 85 (PNMOF-1) or $100^{\circ} \mathrm{C}$ (PNMOF-2 and 3) for 1 day, in the presence of cross-linked copolymers, or as controls, polydivinylbenzene or polypropylene. The microtiter plates were sealed with polyethylene plugs and pressed firmly between aluminum plates to prevent solvent loss upon heating. Each experiment was repeated 8 times within each trial, and at least 2 trials were carried out to gauge reproducibility. Only consistently reproduced results are reported. The same reactions lead exclusively to MOF-5, IRMOF-2, or IRMOF-3 when performed with the appropriate linker in glass vials without the addition of a polymer, under otherwise identical conditions.

X-ray diffraction: PXRD was performed on a Bruker AXS D8 DISCOVER GADDS diffractometer (for combinatorial screening) equipped with a $\mathrm{Cu}_{\mathrm{K} \alpha}$ source $(\lambda=1.5406 \AA)$ and an area detector. Single-crystal X-ray diffraction was performed on a Bruker SMART CCD-based diffractometer with a $\mathrm{Mo}_{\mathrm{K} \alpha}$ source $(\lambda=0.71073 \AA)$. Intensity data were integrated with the SAINT software package and corrected for absorption by using SADABS. The structure was then solved by direct methods and refined by using the SHELXTL (v6.10) software package. All non-hydrogen atoms, with the exception of those of free solvent molecules and severely disordered bound solvent molecules, were refined anisotropically. For PNMOF-1 and 2, hydrogen atoms were generated at idealized positions and refined as riding atoms. For all three structures, the contribution of free solvent molecules was calculated through the use of the SQUEEZE subroutine of the PLATON software suite. ${ }^{[8]}$

Received: December 4, 2005

Published online: March 14, 2006

Keywords: crystal growth - metal-organic frameworks . microporous materials · polymers - supramolecular chemistry

[1] a) H. G. Brittain, Polymorphism in Pharmaceutical Solids, Marcel Dekker, New York, 1999; b) B. Rodríguez-Spong, C. P. Price, A. Jayasankar, A. J. Matzger, N. Rodríguez-Hornedo, Adv. Drug Delivery Rev. 2004, 56, 241-274.

[2] a) D. T. Vodak, K. Kim, L. Iordanidis, P. G. Rasmussen, A. J. Matzger, O. M. Yaghi, Chem. Eur. J. 2003, 9, 4197-4201; b) R. Kitaura, K. Seki, G. Akiyama, S. Kitagawa, Angew. Chem. 2003, 115, 444-447; Angew. Chem. Int. Ed. 2003, 42, 428-431; c) S. Kitagawa, R. Kitaura, S. Noro, Angew. Chem. 2004, 116, $2388-$ 2430; Angew. Chem. Int. Ed. 2004, 43, 2334-2375; d) H. Li, M. Eddaoudi, T. L. Groy, O. M. Yaghi, J. Am. Chem. Soc. 1998, 120, 8571-8572; e) H. Li, M. Eddaoudi, M. O'Keeffe, O. M. Yaghi, Nature 1999, 402, 276-279; f) N. L. Rosi, J. Kim, M. Eddaoudi, B. L. Chen, M. O'Keeffe, O. M. Yaghi, J. Am. Chem. Soc. 2005, 127, 1504-1518.

[3] a) M. Lang, A. L. Grzesiak, A. J. Matzger, J. Am. Chem. Soc. 2002, 124, 14834-14835; b) C. P. Price, A. L. Grzesiak, A. J. Matzger, J. Am. Chem. Soc. 2005, 127, 5512-5517.

[4] In this context, it is interesting to note that recently the growth of MOF-5 from self-assembled monolayers of 16-mercaptohexadecancoic acid on gold has been reported. S. Hermes, F. Schröder, R. Chelmowski, C. Wöll, R. A. Fischer, J. Am. Chem. Soc. 2005, 127, $13744-13745$.

[5] Crystal data: a) PNMOF-1 $\left(\mathrm{Zn}_{3}(\mathrm{BDC})_{3}(\mathrm{DEF})_{2}\right)$ : colorless plate, $0.22 \times 0.11 \times 0.06 \mathrm{~mm}^{3}, 153(2) \mathrm{K}$, monoclinic, space group $C 2 / \mathrm{c}$ (no. 15), $a=25.032(5), \quad b=20.812(4), \quad c=17.738(3) \AA, \quad \beta=$ $114.396(3)^{\circ}, \quad V=8416(3) \AA^{3}, \quad Z=8, \quad \rho_{\text {calcd }}=1.404 \mathrm{~g} \mathrm{~cm}^{-3}, \quad \mu-$ $\left(\mathrm{Mo}_{\mathrm{K} a}\right)=1.757 \mathrm{~mm}^{-1}, F(000)=3624,9740$ unique reflections, $2.64 \leq 2 \theta \leq 57.06^{\circ}, \quad T_{\max }=0.90, \quad T_{\min }=0.70, R_{1}=0.0566, R_{w}=$ 


\section{Zuschriften}

0.1197 ; b) PNMOF-2 $\left(\left(\mathrm{Zn}_{3} \mathrm{O}\right)(\mathrm{BrBDC})_{2}\left(\mathrm{H}_{2} \mathrm{BrBDC}\right)(\mathrm{DEF})_{4}\right)$ : colorless block, $0.22 \times 0.18 \times 0.13 \mathrm{~mm}^{3}, 153(2) \mathrm{K}$, monoclinic, space group $C 2 / c$ (no. 15), $a=43.028(13), b=13.000(4), c=$ 22.077(7) $\AA, \quad \beta=114.964(6)^{\circ}, \quad V=11195(6) \AA^{3}, \quad Z=8, \quad \rho_{\text {calcd }}=$ $1.410 \mathrm{~g} \mathrm{~cm}^{-1}, \quad \mu\left(\mathrm{Mo}_{\mathrm{Ka}}\right)=3.118 \mathrm{~mm}^{-1}, \quad F(000)=4588, \quad 11414$ unique reflections, $2.08 \leq 2 \theta \leq 52.74^{\circ}, T_{\max }=0.67, T_{\min }=0.53$, $\left.R_{l}=0.1411, R_{w}=0.3913 ; \mathrm{c}\right)$ PNMOF-3 $\left(\mathrm{Zn}_{4}\left(\mathrm{NH}_{2} \mathrm{BDC}\right)_{3}\left(\mathrm{NO}_{3}\right)_{2}-\right.$ $\left.\left(\mathrm{H}_{2} \mathrm{O}\right)_{2}\right)$ : orange plate, $0.43 \times 0.17 \times 0.14 \mathrm{~mm}^{3}, 258(2) \mathrm{K}$, hexagonal, space group $P 6 / \mathrm{mmm}$ (no. 191), $a=18.3385(7), \quad c=$ 14.2657(10) $\AA, V=4154.8(4) \AA^{3}, Z=4, \rho_{\text {calcd }}=0.376 \mathrm{~g} \mathrm{~cm}^{-3}, \mu$ $\left(\mathrm{Mo}_{\mathrm{Ka}}\right)=0.589 \mathrm{~mm}^{-1}, \quad F(000)=459,1914$ unique reflections, $3.84 \leq 2 \theta \leq 55.18^{\circ}, \quad T_{\max }=0.92, \quad T_{\min }=0.79, \quad R_{l}=0.0959, \quad R_{w}=$ 0.2686. CCDC-286534 (PNMOF-1), CCDC-286535 (PNMOF-2), and CCDC-286536 (PNMOF-3) contain the supplementary crystallographic data for this paper. These data can be obtained free of charge from The Cambridge Crystallographic Data Centre via www.ccdc.cam.ac.uk/data_request/cif.

[6] M. Eddaoudi, J. Kim, N. Rosi, D. Vodak, J. Wachter, M. O'Keefe, O. M. Yaghi, Science 2002, 295, 469-472.

[7] This value is the distance across the channel from aromatic carbon to aromatic carbon (18.3 $\AA$ ) minus two carbon van der Waals radii (each $1.70 \AA$ A ). A. Bondi, J. Phys. Chem. 1964, 68, $441-451$.

[8] A. L. Spek, Acta Crystallogr. Sect. A 1990, 46, C34. 